\title{
Some general Baskakov type operators
}

\author{
Adrian D. Indrea and Ovidiu T. Pop
}




\title{
SOME GENERAL BASKAKOV TYPE OPERATORS
}

\author{
ADRIAN D. INDREA AND OVIDIU T. POP
}

Received 24 September, 2013

\begin{abstract}
A general class of linear positive operators which generalizes Baskakov's operator is consrtucted. The operators of this type which preserve exactly two test functions from the set $\left\{e_{0}, e_{1}, e_{2}\right\}$ are determined in each case, and for the operators obtained, we give their approximation theorem, convergence theorem and Voronovskaja-type theorem.
\end{abstract}

2010 Mathematics Subject Classification: 41A36; 26D15

Keywords: Baskakov type operators, modulus of continuity, approximation and convergence theorem, Voronovskaja-type theorem

\section{INTRODUCTION}

Let $\mathbb{N}$ be a set of positive integers and $\mathbb{N}_{0}=\mathbb{N} \cup\{0\}$.

In [6], J. P. King constructed and studied general operators which generalizes the classical Berstein operators. Some King-type operators were studied in [3-6], [8,9].

In 1957, V. A. Baskakov [2], for $m \in \mathbb{N}$ has introduced the linear positive operator

$$
\left(V_{m} f\right)(x)=(1+x)^{-m} \sum_{k=0}^{\infty}\left(\begin{array}{c}
m+k-1 \\
k
\end{array}\right)\left(\frac{x}{1+x}\right)^{k} f\left(\frac{k}{m}\right)
$$

defined for any $f \in C_{2}([0,+\infty))=\left\{f \in C([0,+\infty)) \mid \lim _{x \rightarrow \infty} \frac{f(x)}{1+x^{2}}<+\infty\right\}$ and $x \in[0,+\infty)$. He proved that if $f \in C_{2}([0,+\infty))$ then $V_{m} f \longrightarrow f$ uniform on any compact $[a, b] \subset[0,+\infty)$. Note that the operators (1.1) preserve the test functions $e_{0}$ and $e_{1}$. Generalizations of the operators (1.1) were introduced by M.A.Özarslan, G.Duman and N.I.Mahmudov in [10] by the form

$$
\left(T_{m} f\right)(x)=\sum_{k=0}^{\infty}\left(\begin{array}{c}
m+k-1 \\
k
\end{array}\right)\left(u_{m}(x)\right)^{k}\left(1+u_{m}(x)\right)^{-m-k} f\left(\frac{k}{m}\right)
$$

for $m \in \mathbb{N}, x \in[0,+\infty)$, and they show that if $u_{m}(x) \longrightarrow x$ on a compact $[a, b] \subset$ $[0,+\infty)$, then $T_{m} f \longrightarrow f$ uniform on $[a, b]$ for all $f \in C_{2}([0,+\infty))$.

(c) 2014 Miskolc University Press 
A similar result was obtained in [9] by L. Rempulska and K. Tomczak for the case in which the modified operators of Baskakov type preserve the test functions $e_{0}$ and $e_{2}$.

In this paper, we introduce a general class of linear positive operators. We determine the operators of the general class which preserve only two test functions $e_{0}$ and $e_{1}$ or $e_{0}$ and $e_{2}$ or $e_{1}$ and $e_{2}$.

In all these cases we give approximation properties, convergence theorems and Voronovskaja-type theorems.

The paper is organized as follows. In Section 2 we recall some results obtained by O.T.Pop in [7] which are essentially used for obtaining the main results of the paper. Section 3 is devoted to the construction of the general class of linear and positive operators defined by infinite sum, which we announced in the start. For the constructed class we establish a convergence theorem and Voronovskaja type theorem. In Section 4 we prove that in the general class constructed in Section 3 exists a unique operator which preserve the test functions $e_{0}$ and $e_{1}$, the classical Baskakov operator. In Section 5 we obtain a King type operator, which is an operator that preserves the test functions $e_{0}$ and $e_{2}$ defined on semiaxis $[0,+\infty)$. We find here a result due the L. Rempulska and K. Tomczak [9].

Finally, in Section 6, we determine the operators from the general class which preserve the test function $e_{1}$ and $e_{2}$.

\section{PRELIMINARIES}

In this section we recall some results from [7], which we shall use in the present paper. Let $I, J$ be real intervals with the property $I \cap J$ is a nonempty interval. For any $m, k \in \mathbb{N}_{0}, m \neq 0$, we consider the functions $\varphi_{m, k}: J \longrightarrow \mathbb{R}$, with the property that $\varphi_{m, k}(x) \geq 0$, for any $x \in J$ and the linear positive functionals $A_{m, k}: E(I) \longrightarrow \mathbb{R}$.

For any $m \in \mathbb{N}$ we define the operator $L_{m}: E(I) \longrightarrow F(J)$, by

$$
\left(L_{m} f\right)(x)=\sum_{k=0}^{\infty} \varphi_{m, k}(x) A_{m, k}(f),
$$

where $E(I)$ is a linear space of real valued functions defined on $I$, for which the operators (2.1) are convergent and $F(J)$ is a subset of real valued functions defined on $J$.

Remark 1. The operators $\left(L_{m}\right)_{m \in \mathbb{N}}$ are linear and positive on $E(I \cap J)$.

For $m \in \mathbb{N}$ and $i \in \mathbb{N}_{0}$, we define $T_{m, i}$ by

$$
\left(T_{m, i} L_{m}\right)(x)=m^{i}\left(L_{m} \psi_{x}^{i}\right)(x)=m^{i} \sum_{k=0}^{\infty} \varphi_{m, k}(x) A_{m, k}\left(\psi_{x}^{i}\right)
$$

for any $x \in I \cap J$, where $\psi_{x}: I \longrightarrow \mathbb{R}, \psi_{x}(t)=t-x$. 
In what follows $s \in \mathbb{N}_{0}$ is even and we assume that the following condition: there exist the smallest $\alpha_{s}, \alpha_{s+2} \in[0,+\infty)$, so that

$$
\lim _{m \rightarrow \infty} \frac{\left(T_{m, j} L_{m}\right)(x)}{m^{\alpha_{j}}}=B_{j}(x) \in \mathbb{R}
$$

for any $x \in I \cap J$ and $j \in\{s, s+2\}$,

$$
\alpha_{s+2}<\alpha_{s}+2
$$

hold.

Theorem 1 ([7]). Let $f \in E(I)$ be a function. If $x \in I \cap J$ and $f$ is s times differentiable in a neighborhood of $x, f^{(s)}$ is continuous on $x$, then

$$
\lim _{m \rightarrow \infty} m^{s-\alpha_{s}}\left(\left(L_{m} f\right)(x)-\sum_{i=0}^{s} \frac{f^{(i)}(x)}{m^{i} i !}\left(T_{m, i} L_{m}\right)(x)\right)=0 .
$$

Assume that $f$ is $s$ times differentiable on $I$. Let $K \subset I \cap J$ be a compact interval. For there one we assume that exist $m(s) \in \mathbb{N}$ and constant $k_{j} \in \mathbb{R}$ depending on $K$, such that for $m \geq m(s)$ and $x \in K$ the following relation

$$
\frac{\left(T_{m, j} L_{m}\right)(x)}{m^{\alpha_{j}}} \leq k_{j}, j \in\{s, s+2\}
$$

holds.

Following [7], the convergence expressed by (2.5) is uniform on $K$ and

$$
\begin{gathered}
m^{s-\alpha_{s}}\left|\left(L_{m} f\right)(x)-\sum_{i=0}^{s} \frac{f^{(i)}(x)}{m^{i} i !}\left(T_{m, i} L_{m}\right)(x)\right| \leq \\
\leq \frac{1}{s !}\left(k_{s}+k_{s+2}\right) \omega\left(f^{(s)} ; \frac{1}{\sqrt{m^{2+\alpha_{s}-\alpha_{s+2}}}}\right),
\end{gathered}
$$

for any $x \in K, m \geq m(s)$, where $\omega(f ; \delta)$ denotes the modulus of continuity of the function $f$.

In the following, we use the identity

$$
(1+x)^{-m}=\sum_{k=0}^{\infty}(-1)^{k}\left(\begin{array}{c}
m+k-1 \\
k
\end{array}\right) x^{k}
$$

where $x \geq 0$ and $m \in \mathbb{N}$.

By differentiating the relation (2.8) and multiplying with $\frac{x}{m}$, we obtain

$$
-x(1+x)^{-m-1}=\sum_{k=0}^{\infty}(-1)^{k}\left(\begin{array}{c}
m+k-1 \\
k
\end{array}\right) x^{k} \frac{k}{m} .
$$


Similarly, differentiating the relation (2.9) and multiplying with $\frac{x}{m}$ we get

$$
\frac{x}{m}(m x-1)(1+x)^{-m-2}=\sum_{k=0}^{\infty}(-1)^{k}\left(\begin{array}{c}
m+k-1 \\
k
\end{array}\right) x^{k}\left(\frac{k}{m}\right)^{2},
$$

where $x \geq 0$ and $m \in \mathbb{N}$.

\section{THE CONSTRUCTION OF A GENERAL LINEAR AND POSITIVE OPERATORS DEFINED BY INFINITE SUM}

Let $m_{0} \in \mathbb{N}$ be given, $\mathbb{N}_{1}=\left\{m \in \mathbb{N} \mid m \geq m_{0}\right\}$, the functions $\alpha_{m}: J \longrightarrow \mathbb{R}$ and $\beta_{m}: J \longrightarrow \mathbb{R}$ such that $\alpha_{m}(x)>0, \beta_{m}(x)>0, \beta_{m}(x)-\alpha_{m}(x)>0$ for any $x \in J$ and any $m \in \mathbb{N}_{1}$.

We define the operators of the following form

$$
\left(P_{m} f\right)(x)=\sum_{k=0}^{\infty}\left(\begin{array}{c}
m+k-1 \\
k
\end{array}\right) \alpha_{m}^{k}(x) \beta_{m}^{-m-k}(x) f\left(\frac{k}{m}\right),
$$

for any $m \in \mathbb{N}_{1}, x \in J$ and $f \in E([0,+\infty))$, where $E([0, \infty))$ is a linear space of real valued functions defined on $[0, \infty)$, for which the operators defined by (3.1) are convergent.

If in (2.8)-(2.10), we substitute $x$ by $-\frac{\alpha_{m}(x)}{\beta_{m}(x)}$, we obtain

$$
\begin{gathered}
\left(\beta_{m}(x)-\alpha_{m}(x)\right)^{-m}=\sum_{k=0}^{\infty}\left(\begin{array}{c}
m+k-1 \\
k
\end{array}\right)\left(\alpha_{m}(x)\right)^{k}\left(\beta_{m}(x)\right)^{-m-k} \\
\alpha_{m}(x)\left(\beta_{m}(x)-\alpha_{m}(x)\right)^{-m-1}=\sum_{k=0}^{\infty}\left(\begin{array}{c}
m+k-1 \\
k
\end{array}\right)\left(\alpha_{m}(x)\right)^{k}\left(\beta_{m}(x)\right)^{-m-k} \frac{k}{m} \\
\frac{1}{m} \alpha_{m}(x)\left(m \alpha_{m}(x)+\beta_{m}(x)\right)\left(\beta_{m}(x)-\alpha_{m}(x)\right)^{-m-2}= \\
=\sum_{k=0}^{\infty}\left(\begin{array}{c}
m+k-1 \\
k
\end{array}\right)\left(\alpha_{m}(x)\right)^{k}\left(\beta_{m}(x)\right)^{-m-k}\left(\frac{k}{m}\right)^{2}, x \in J, m \in \mathbb{N} .
\end{gathered}
$$

We impose the condition

$$
\left(P_{m} e_{0}\right)(x)=1+u_{m}(x),
$$

for any $m \in \mathbb{N}_{1}$ and any $x \in J$, where $u_{m}: J \longrightarrow \mathbb{R}, u_{m}(x)>-1$.

From (3.1), (3.2) and (3.5) follows the equality

$$
\beta_{m}(x)-\alpha_{m}(x)=\left(1+u_{m}(x)\right)^{-\frac{1}{m}}
$$

for any $m \in \mathbb{N}_{1}$ and any $x \in J$. 
Let us to impose the condition

$$
\left(P_{m} e_{1}\right)(x)=x+v_{m}(x)
$$

for any $m \in \mathbb{N}_{1}$ and any $x \in J$, where $v_{m}: J \longrightarrow \mathbb{R}, v_{m}(x)>-x$.

Taking (3.1), (3.3) and (3.7) into account, we get

$$
\alpha_{m}(x)\left(\beta_{m}(x)-\alpha_{m}(x)\right)^{-m-1}=x+v_{m}(x), m \in \mathbb{N}_{1}, x \in J .
$$

From (3.6) and (3.8) it follows

$$
\alpha_{m}(x)=\frac{x+v_{m}(x)}{1+u_{m}(x)}\left(1+u_{m}(x)\right)^{-\frac{1}{m}}
$$

and

$$
\beta_{m}(x)=\left(1+\frac{x+v_{m}(x)}{1+u_{m}(x)}\right)\left(1+u_{m}(x)\right)^{-\frac{1}{m}},
$$

$m \in \mathbb{N}_{1}, x \in J$.

Taking (3.9) and (3.10) into account, the operator (3.1) becomes

$$
\begin{gathered}
\left(P_{m} f\right)(x)=\left(1+u_{m}(x)\right) \sum_{k=0}^{\infty}\left(\begin{array}{c}
m+k-1 \\
k
\end{array}\right)\left(\frac{x+v_{m}(x)}{1+u_{m}(x)}\right)^{k} . \\
\cdot\left(1+\frac{x+v_{m}(x)}{1+u_{m}(x)}\right)^{-m-k} f\left(\frac{k}{m}\right),
\end{gathered}
$$

$m \in \mathbb{N}_{1}, x \in J, f \in E([0,+\infty))$.

From (3.1) and (3.4), we have

$$
\left(P_{m} e_{2}\right)(x)=\frac{x+v_{m}(x)}{m}\left((m+1) \frac{x+v_{m}(x)}{1+u_{m}(x)}+1\right),
$$

for any $m \in \mathbb{N}_{1}$ and any $x \in J$.

Next $\left(P_{m} \psi_{x}^{2}\right)(x)=\left(P_{m} e_{2}\right)(x)-2 x\left(P_{m} e_{1}\right)(x)+x^{2}\left(P_{m} e_{0}\right)(x)$ and taking (3.5), (3.7) and (3.12) into account we get

$$
\left(P_{m} \psi_{x}^{2}\right)(x)=\frac{m\left(v_{m}(x)-x u_{m}(x)\right)^{2}+\left(x+v_{m}(x)\right)^{2}+\left(1+u_{m}(x)\right)\left(x+v_{m}(x)\right)}{m\left(1+u_{m}(x)\right)}
$$

for any $m \in \mathbb{N}_{1}$ and any $x \in J$.

Coming back to Theorem 1 , for the operators (3.1), we have $I=[0,+\infty), E(I)=$ $C_{2}([0,+\infty))$

$$
\varphi_{m, k}(x)=\left(1+u_{m}(x)\right)\left(\begin{array}{c}
m+k-1 \\
k
\end{array}\right)\left(\frac{x+v_{m}(x)}{1+u_{m}(x)}\right)^{k}\left(1+\frac{x+v_{m}(x)}{1+u_{m}(x)}\right)^{-m-k}
$$

and

$$
A_{m, k}(f)=f\left(\frac{k}{m}\right)
$$


for any $m \in \mathbb{N}_{1}, x \in J$ and $f \in C_{2}([0,+\infty))$.

In the following, let $K \subset I \cap J$ be a compact interval. that

We suppose that there exists the sequences $\left(a_{m}(K)\right)_{m \in \mathbb{N}_{1}},\left(b_{m}(K)\right)_{m \in \mathbb{N}_{1}}$, so

$$
\begin{gathered}
\lim _{m \longrightarrow \infty} a_{m}(K)=\lim _{m \longrightarrow \infty} b_{m}(K)=0, \\
\left|u_{m}(x)\right| \leq a_{m}(K), \\
\left|v_{m}(x)\right| \leq b_{m}(K),
\end{gathered}
$$

for any $m \in \mathbb{N}_{1}$ and any $x \in K$.

In what follows, let us suppose that the following equality

$$
\lim _{m \longrightarrow \infty} m\left(v_{m}(x)-x u_{m}(x)\right)=l(x)
$$

holds for any $x \in J$, where $l: J \longrightarrow \mathbb{R}$ is a bounded function on $K$.

Remark 2. From (3.16) - (3.18) it results that if

$$
\lim _{m \longrightarrow \infty} u_{m}(x)=\lim _{m \longrightarrow \infty} v_{m}(x)=0, x \in K,
$$

then

$$
\begin{gathered}
\lim _{m \longrightarrow \infty} m\left(v_{m}(x)-x u_{m}(x)\right)^{2}=\lim _{m \rightarrow \infty} m\left(v_{m}(x)-x u_{m}(x)\right) . \\
\cdot \lim _{m \rightarrow \infty}\left(v_{m}(x)-x u_{m}(x)\right)=0 .
\end{gathered}
$$

This Remark 2 implies that there exist $m_{1} \in \mathbb{N}$ such that

$$
\left(m\left(v_{m}(x)-x u_{m}(x)\right)^{2} \leq 1, m \in \mathbb{N}_{1}, m \geq m_{1}, x \in K .\right.
$$

Let us denote

$$
\begin{aligned}
& M_{1}(K)=\sup \left\{a_{m}(K) \mid m \in \mathbb{N}_{1}\right\}, \\
& M_{2}(K)=\sup \left\{b_{m}(K) \mid m \in \mathbb{N}_{1}\right\} .
\end{aligned}
$$

Now, let $\mathbb{N}_{2}=\left\{m \in \mathbb{N} \mid m \geq \max \left(m_{0}, m_{1}\right)\right\}$.

According to Theorem 1 one obtains $\alpha_{0}=0, \alpha_{2}=1,\left(T_{m, 0} P_{m}\right)(x)=\left(P_{m} e_{0}\right)(x)$, for any $m \in \mathbb{N}_{1}$ and any $x \in K$.

From (3.16) one arrives at

$$
\lim _{m \rightarrow \infty}\left(T_{m, 0} P_{m}\right)(x)=1=B_{0}(x), x \in K .
$$

Consequently we get that exists $m(0) \in \mathbb{N}$ such that

$$
\left(T_{m, 0} P_{m}\right)(x)=1+u_{m}(x) \leq 1+M_{1}(K)=k_{0}(K)
$$

holds for any $m \geq \max \left(m_{0}, m(0)\right)$ and $x \in K$.

We have $\left(T_{m, 2} P_{m}\right)(x)=m^{2}\left(P_{m} \psi_{x}^{2}\right)(x), m \in \mathbb{N}_{1}, x \in J$. Taking (3.13), (3.19) and (3.20) into account, we get

$$
\lim _{m \longrightarrow \infty} \frac{\left(T_{m, 2} P_{m}\right)(x)}{m}=x(1+x)+l(x)=B_{2}(x), x \in K .
$$


Also there exists $m(2) \in \mathbb{N}$ such that

$$
\frac{\left(T_{m, 2} P_{m}\right)(x)}{m} \leq b(1+b)+2=k_{2}(K)
$$

for any $m \geq \max \left(m_{0}, m(2), m_{1}\right)$ and $x \in K$, where $\max K=b$.

Theorem 2. Let $f \in C_{2}([0,+\infty))$. Then

$$
\lim _{m \longrightarrow \infty} P_{m} f=f
$$

uniformly on $K$. There exists $m(0) \in \mathbb{N}, m(0)$ depending on $K$, so that the following inequalities

$$
\begin{gathered}
\left|\left(P_{m} f\right)(x)-\left(1+u_{m}(x)\right) f(x)\right| \leq\left(k_{0}(K)+k_{2}(K)\right) \omega\left(f ; \frac{1}{\sqrt{m}}\right), \\
\left|\left(P_{m} f\right)(x)-f(x)\right| \leq\left|u_{m}(x)\right| \cdot|f(x)|+\left(k_{0}(K)+k_{2}(K)\right) \omega\left(f ; \frac{1}{\sqrt{m}}\right)
\end{gathered}
$$

and

$$
\left|\left(P_{m} f\right)(x)-f(x)\right| \leq a_{m}(K) M(K)+\left(k_{0}(K)+k_{2}(K)\right) \omega\left(f ; \frac{1}{\sqrt{m}}\right)
$$

hold for any $m \in \mathbb{N}_{2}, m \geq m(0)$ and $x \in K$, where

$$
M(K)=\sup \{|f(x)| \mid x \in K\} .
$$

Proof of Theorem 2. Applying the Theorem 1 for $\alpha=0$ yields (3.25) and (3.26). Next, using the inequality $|a-c|-|b-c| \leq|a-b|$, (3.27) follows, and consequently (3.28) holds.

Remark 3. The equations (3.26)-(3.28) are asymptotic formula for a class of approximation processes of King's type (see [1]).

Theorem 3. Let $f \in C_{2}([0,+\infty))$. If $x \in K$, $f$ is two times differentiable in $x$ and $f^{(2)}$ is continuous in $x$, the following relations

$$
\left.\lim _{m \longrightarrow \infty} m\left(\left(P_{m} f\right)(x)-\left(1+u_{m}(x)\right) f(x)\right)=l(x)\right) f^{(1)}(x)+\frac{x(1+x)}{2} f^{(2)}(x)
$$

holds.

Proof of Theorem 3. If $m \in \mathbb{N}_{1}, x \in K$, according Theorem 1 yields

$$
\left(T_{m, 1} P_{m}\right)(x)=m\left(P_{m} \psi_{x}\right)(x)=m\left(\left(P_{m} e_{1}\right)(x)-x\left(P_{m} e_{0}\right)(x)\right) .
$$

Applying (3.1) and (3.5) it follows

$$
\left(T_{m, 1} P_{m}\right)(x)=m\left(v_{m}(x)-x u_{m}(x)\right) .
$$

Using Theorem 1 for $s=2$, (3.22), (3.23) and (3.30) one arrives at (3.29).

Remark 4. The relation (3.29) is a Voronovskaja-type theorem (see [11]). 


\section{4. $\left(P_{m}\right)_{m \geq m_{0}}$ OPERATORS PRESERVING TEST FUNCTIONS $e_{0}$ AND $e_{1}$}

In the following, we consider $K=[a, b]$, where $b>0$. In this case $J=[0,+\infty)$ and $m_{0}=1$, then $\mathbb{N}_{1}=\mathbb{N}$. If the operators, $\left(P_{m}\right)_{m \in \mathbb{N}}$ preserve $e_{0}$ and $e_{1}$, we have $P_{m} e_{0}=e_{0}$ and $P_{m} e_{1}=e_{1}$, for any $m \in \mathbb{N}$. Taking (3.5) and (3.7) into account, it results that $u_{m}(x)=v_{m}(x)=0$ and $l(x)=0$ for any $m \in \mathbb{N}$ and any $x \in[0,+\infty)$.

In this case, we get again the classical Baskakov operators. One has $a_{m}([a, b])$ $=b_{m}([a, b])=0$, for any $m \in \mathbb{N}, k_{0}([a, b])=1$ and $k_{2}([a, b])=b(1+b)+2$. Our statements turn into well known results.

Theorem 4 ([2]). Let $f \in C_{2}([0,+\infty))$ one has

$$
\lim _{m \rightarrow \infty} P_{m} f=f
$$

uniformly on any compact interval $[a, b] \subset \mathbb{R}_{+}$and then exists $m(0) \in \mathbb{N}, m(0)$ depending on $b$ so that

$$
\left|\left(P_{m} f\right)(x)-f(x)\right| \leq\left(3+b+b^{2}\right) \omega\left(f ; \frac{1}{\sqrt{m}}\right), m \in \mathbb{N}_{2}, m \geq m(0), x \in[a, b] .
$$

Theorem 5 ([2]). Let $f \in C_{2}([0,+\infty))$. If $x \in[a, b], f$ is two times differentiable in $x$ and $f^{(2)}$ is continuous in $x$, then

$$
\lim _{m \longrightarrow \infty} m\left(\left(P_{m} f\right)(x)-f(x)\right)=\frac{x(1+x)}{2} f^{(2)}(x) .
$$

5. $\left(P_{m}\right)_{m \geq m_{0}}$ OPERATORS PRESERVING THE TEST FUNCTIONS $e_{0}$ AND $e_{2}$

In this case $J=[0,+\infty)$ and $m_{0}=1$, then $\mathbb{N}_{1}=\mathbb{N}$. Because $P_{m} e_{0}=e_{0}$ and $P_{m} e_{2}=e_{2}$ for any $m \in \mathbb{N}$, taking (3.5) into account, it follows $u_{m}(x)=0$, for any $m \in \mathbb{N}$ and any $x \in[0,+\infty)$.

By using (3.12) yields

$$
(m+1)\left(x+v_{m}(x)\right)^{2}+\left(x+v_{m}(x)\right)-m x^{2}=0
$$

for any $m \in \mathbb{N}$ and any $x \in[0,+\infty)$.

From (5.1) we get $v_{m}(x)=\frac{\sqrt{4 m(m+1) x^{2}+1}-1}{2(m+1)}-x$, for any $m \in \mathbb{N}$ and any $x \in$ $[0,+\infty)$, and then the operators from (3.8) become

$$
\begin{gathered}
\left(P_{m} f\right)(x)=\sum_{k=0}^{\infty}\left(\begin{array}{c}
m+k-1 \\
k
\end{array}\right)\left(\frac{\sqrt{4 m(m+1) x^{2}+1}-1}{2(m+1)}\right)^{k} . \\
\cdot\left(1+\frac{\sqrt{4 m(m+1) x^{2}+1}-1}{2(m+1)}\right)^{-m-k} f\left(\frac{k}{m}\right),
\end{gathered}
$$

$m \in \mathbb{N}, x \in[0,+\infty), f \in C_{2}([0,+\infty))$.

So we came across the results obtained by L. Rempulska and K. Tomczak in [9]. 
Lemma 1. We have that

$$
v_{m}(x) \leq \frac{\sqrt{4 m(m+1) a^{2}+1}-1}{2(m+1)}-a, m \in \mathbb{N}, x \in K=[a, b]
$$

and

$$
\frac{\sqrt{4 m(m+1) a^{2}+1}-1}{2(m+1)}-a \leq \sqrt{\frac{1}{2} a^{2}+\frac{1}{16}}-a, m \in \mathbb{N} .
$$

Proof of Lemma 1. Since the function $v_{m}$ is decreasing on $[a, b]$, it gets the maximum value in $a$ and (5.3) follows. By direct computation, (5.4) is obtained.

Lemma 2. The following relation

$$
\lim _{m \longrightarrow \infty} m v_{m}(x)=-\frac{1+x}{2}
$$

holds, where $x \in K$.

Proof of Lemma 2. We have

$$
\begin{gathered}
\lim _{m \longrightarrow \infty} m v_{m}(x)=\lim _{m \longrightarrow \infty} \frac{m}{2(m+1)}\left(-1+\sqrt{4 m(m+1) x^{2}+1}-2(m+1) x\right)= \\
=\frac{1}{2}\left(-1+\lim _{m \rightarrow \infty} \frac{-4 m x^{2}-4 x^{2}+1}{\sqrt{4 m(m+1) x^{2}+1}+2(m+1) x}\right)
\end{gathered}
$$

and (5.5) follows.

According to the notations from Section 3, taking Lemma 1 and Lemma 2 into account we have $a_{m}([a, b])=0$, for any $m \in \mathbb{N}, b_{m}([a, b])$

$=\frac{\sqrt{4 m(m+1) a^{2}+1}-1}{2(m+1)}-a, l(x)=-\frac{1+x}{2}$, for any $m \in \mathbb{N}$, any $x \in[a, b], b_{m}([a, b]) \leq$ $\sqrt{\frac{1}{2} a^{2}+\frac{1}{16}}-a=M_{2}([a, b])$, for any $m \in \mathbb{N}$ and then $M_{1}([a, b])=0, k_{0}([a, b])=1$, $k_{2}([a, b])=b(1+b)+2$.

As consequences of Theorem 2 we get

Theorem 6. For any $f \in C_{2}([0,+\infty))$ it follows

$$
\lim _{m \rightarrow \infty} P_{m} f=f
$$

uniformly on compact $[a, b]$ and there exists $m(0) \in \mathbb{N}, m(0)$ depending on $b$, so that

$$
\left|\left(P_{m} f\right)(x)-f(x)\right| \leq(3+b(1+b)) \omega\left(f ; \frac{1}{\sqrt{m}}\right), m \in \mathbb{N}_{2}, m \geq m(0), x \in[a, b] .
$$

Theorem 7. Let $f \in C_{2}([0,+\infty))$. If $x \in[a, b]$, $f$ is two times differentiable in $x$ and $f^{(2)}$ is continuous in $x$, then

$$
\lim _{m \longrightarrow \infty} m\left(\left(P_{m} f\right)(x)-f(x)\right)=-\frac{1+x}{2} f^{(1)}(x)+\frac{x(1+x)}{2} f^{(2)}(x) .
$$


Proof of Theorem 7. Taking Lemma 2 into account and applying (3.29), (5.8) is obtained.

\section{6. $\left(P_{m}\right)_{m \geq m_{0}}$ OPERATORS PRESERVING THE TEST FUNCTIONS $e_{1}$ AND $e_{2}$}

In this case $m_{0} \in \mathbb{N}, m_{0} \geq 2$ is a fixed number and $J=\left[\frac{1}{m_{0}-1},+\infty\right)$. If $P_{m} e_{1}=$ $e_{1}$, for any $m \in \mathbb{N}_{1}$, yields $v_{m}(x)=0$, for any $m \in \mathbb{N}_{1}$ and any $x \in\left[\frac{1}{m_{0}-1},+\infty\right)$. For $x \geq \frac{1}{m_{0}-1}$, we have $\frac{m x-1}{x+1} \geq \frac{m-m_{0}+1}{m_{0}}$ because the function $\frac{x+1}{m x-1}$ is decreasing on $\left[\frac{1}{m_{0}-1},+\infty\right)$, from where $\frac{m x-1}{x+1}>0$ for any $m \in \mathbb{N}_{1}$ and any $x \in\left[\frac{1}{m_{0}-1},+\infty\right)$. Taking (3.12) into account, from $P_{m} e_{1}=e_{1}$ and $P_{m} e_{2}=e_{2}$ for any $m \in \mathbb{N}_{1}$, we have $\frac{m+1}{m} \frac{x^{2}}{1+u_{m}(x)}+\frac{x}{m}=x^{2}$, for any $x \in\left[\frac{1}{m_{0}-1},+\infty\right)$, from where

$$
u_{m}(x)=\frac{x+1}{m x-1}, m \in \mathbb{N}_{1}, x \in\left[\frac{1}{m_{0}-1},+\infty\right) .
$$

Then the operators from (3.11) become

$$
=\frac{(m+1) x}{m x-1} \sum_{k=0}^{\infty}\left(\begin{array}{c}
m+k-1 \\
k
\end{array}\right)\left(\frac{m x-1}{m+1}\right)^{k}\left(1+\frac{x-1}{m+1}\right)^{-m-k} f\left(\frac{k}{m}\right)
$$

for $m \in \mathbb{N}_{1}, x \in\left[\frac{1}{m_{0}-1},+\infty\right)$ and $f \in C_{2}([0,+\infty))$.

According to the notations from Section 3, we have $b_{m}\left(\left[\frac{1}{m_{0}-1}, b\right]\right)=0, l(x)=$ $-1-x$, for any $m \in \mathbb{N}_{1}$, and because the function $u_{m}(x)=\frac{x+1}{m x-1}$ is decreasing on $\left[\frac{1}{m_{0}-1},+\infty\right)$, we get that

$$
u_{m}(x) \leq \frac{m_{0}}{m-m_{0}+1}=a_{m}\left(\left[\frac{1}{m_{0}-1}, b\right]\right)
$$

for any $x \in\left[\frac{1}{m_{0}-1}, b\right)$ and $M_{2}\left(\left[\frac{1}{m_{0}-1}, b\right]\right)=0$. Then $k_{0}=1+m_{0}, k_{2}=b(1+$ b) +2 and $M_{1}\left(\left[\frac{1}{m_{0}-1}, b\right]\right)=m_{0}$.

Theorem 8. For any $f \in C_{2}([0,+\infty))$ it follows

$$
\lim _{m \rightarrow \infty} P_{m} f=f
$$

uniformly on the compact $\left[\frac{1}{m_{0}-1}, b\right]$ and there exists $m(0) \in \mathbb{N}$ depending on $b$, such that

$$
\left|\left(P_{m} f\right)(x)-f(x)\right| \leq \frac{m_{0}}{m-m_{0}+1} M\left(\left[\frac{1}{m_{0}-1}, b\right]\right)+
$$




$$
+\left(3+m_{0}+b(1+b)\right) \omega\left(f ; \frac{1}{\sqrt{m}}\right)
$$

for any $m \in \mathbb{N}_{2}, m \geq m(0)$ and $x \in\left[\frac{1}{m_{0}-1}, b\right]$, where

$$
M\left(\left[\frac{1}{m_{0}-1}, b\right]\right)=\sup \left\{|f(x)| \mid x \in\left[\frac{1}{m_{0}-1}, b\right]\right\} .
$$

Proof of Theorem 8. It results immediately from Theorem 2.

Theorem 9. Let $f \in C_{2}\left([0,+\infty)\right.$. If $x \in\left[\frac{1}{m_{0}-1}, b\right]$, $f$ is two times differentiable in $x$ and $f^{(2)}$ is continuous in $x$, then

$$
\lim _{m \rightarrow \infty} m\left(\left(P_{m} f\right)(x)-f(x)\right)=\frac{1+x}{x} f(x)-(1+x) f^{(1)}(x)+\frac{x(1+x)}{2} f^{(2)}(x) .
$$

Proof of Theorem 9. We have $\lim _{m \rightarrow \infty} m u_{m}(x)=\frac{1+x}{x}, l(x)=-1-x$, for any $x \in\left[\frac{1}{m_{0}-1}, b\right]$ and taking (3.29) into account, follows (6.5).

\section{REFERENCES}

[1] O. Agratini, "An asymptotic formula for a class of approximation processes of King's type," Studia Sci. Math Hungar, vol. 47, no. 4, pp. 435-444, 2010.

[2] V. A. Baskakov, "An example of sequence of linear positive operators in the space of continuous functions," Dokl. Akad. Nouk. SSSR, vol. 113, pp. 249-251, 1957.

[3] P. I. Braica, O. T. Pop, and A. D. Indrea, "About a King-type operator," Appl. Math. Inf. Sci., vol. 6, no. 1, pp. 145-148, 2012.

[4] H. Gonska and P. Pitul, "Remarks on an article of J. P. King," Comment Math. Univ. Carolin, vol. 46, no. 4, pp. 645-666, 2005.

[5] A. D. Indrea, "A particular class of linear and positive Stancu-type operators," Acta Univ. Apulensis, no. 31, pp. 249-256, 2012.

[6] J. P. King, "Positive linear operators which preserve $x^{2}$," Acta. Math. Hungar, vol. 3, no. 99, pp. 203-208, 2003.

[7] O. T. Pop, "About some linear and positive operators defined by infinite sum," Dem. Math., vol. XXXIX, no. 2, pp. -377-388, 2006.

[8] O. T. Pop, A. D. Indrea, and P. I. Braica, "Durrmeyer operators of King-type," Annals of the University of Craiova, vol. 39, no. 2, pp. 288-298, 2012.

[9] L. Rempulska and K. Tomczak, "Approximation by certain linear operators preserving $x^{2}$," Furk. J. Math., vol. 33, pp. 273-281, 2009.

[10] M. A. Özarslan, G. Duman, and N. I. Mahmudov, "Local approximation properties of modified Baskakov operators," Springer Basel A.G. Math, vol. 59, pp. 1-11, 2011.

[11] E. Voronovskaja, "Determination de la forme asumptotique d'approximation des fonctions par les polynômes de S. N. Berstein,” C.R. Acad. Sci. URSS, p. 7985, 1932. 
Authors' addresses

Adrian D. Indrea

Tehnological High School of Tarna Mare 22 tăşnadului Street 440114 Satu Mare, Romania E-mail address: adrian.indrea@yahoo.com

Ovidiu T. Pop

National College "Mihai Eminescu" 5 Mihai Eminescu Street 440014 Satu Mare, Romania E-mail address: ovidiutiberiulyahoo.com 